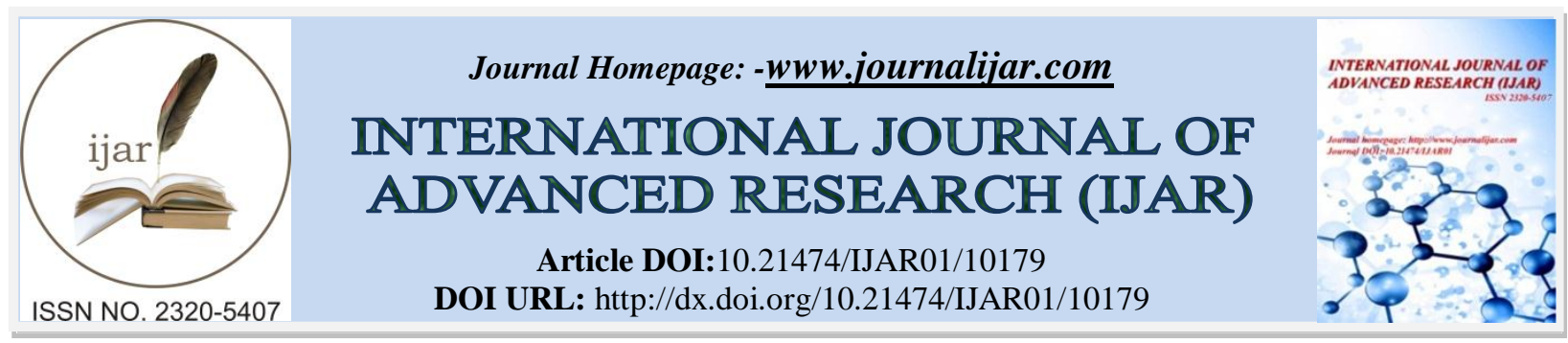

RESEARCH ARTICLE

\title{
MODULATION OF VARIOUS BIOMARKERS UNDER THE EFFECT OF VARIOUS DRUGS DURING ORTHODONTIC TOOTH MOVEMENT
}

\author{
Dr. Nikita Gulati, Dr. Monis Raza and Dr. Divya Shetty
}

\section{Manuscript Info}

Manuscript History

Received: 05 October 2019

Final Accepted: 07 November 2019

Published: December 2019

Key words:-

Biomarker, Drug, Tooth

\begin{abstract}
The biological processes that come into play during orthodontic tooth movement (OTM) have been shown to be influenced by a variety of pharmacological agents. The effects of such agents are of particular relevance to the clinician as the rate of tooth movement can be accelerated or reduced as a result. The aim of the present review is to summarize the knowledge of these biomarkers which could be used in accelerating or manipulating orthodontic treatment under the effect of routinely prescribed drugs which impact and modulate these biomarkers and in turn the orthodontic tooth movement.
\end{abstract}

Copy Right, IJAR, 2019,. All rights reserved.

\section{Introduction:-}

Orthodontic treatment is based on the principle that application of force to a tooth which is transmitted to the neighboring investing tissues, leads to cellular and biochemical changes within these tissues which allow for mechanical alterations and contribute to the tooth movement. ${ }^{1}$ The obvious changes which occur in the tooth supporting apparatus are like resorption of the bone on the pressure side of the socket wall, ahead of the advancing tooth, while deposition of bone on the tension side of the socket maintains a progressively advancing socket wall behind the moving tooth. Prospective biological markers can be collected from different tissue samples, such as gingival crevicular fluid, saliva and serum in order to study their role in orthodontic tooth movement. Several potential biomarkers representing these biological changes during specific phenomenon, such as, bone remodelling (formation and resorption), inflammation, and root resorption have been studied. ${ }^{2}$ The aim of the present review is to summarize the knowledge of these biomarkers which could be used in accelerating or manipulating orthodontic treatment under the effect of routinely prescribed drugs which impact and modulate these biomarkers and in turn the orthodontic tooth movement.

\section{Biomarkers Expressed During Orthodontic Tooth Movement:}

Early phase of orthodontic tooth movement involves an acute inflammatory response characterized by periodontal vasodilatation, and pain sensation is a common reaction by patients subjected to orthodontic forces. Orthodontists have long observed that teeth move at different rates and individuals differ in their response to treatment. A biomarker is a substance that is measured and evaluated objectively as an indicator of normal biologic processes, pathogenic processes, or pharmacologic responses to a therapeutic intervention. ${ }^{1}$

In investigating biomarkers, the rate, amount, and activity of the released substances not only reflect the activity of individual cells but also indicates its systemic activity. A good biomarker demonstrates high specificity and sensitivity. Specific markers are prominent only in orthodontic tooth movement. Sensitive markers should have the ability to inform about the biological condition in terms of periodontal tissue changes and their relationships with the

Corresponding Author:-Dr. Nikita Gulati 
particular phase of tooth movement. Knowledge of the ongoing process can lead to proper mechanical loading and thus shorten the period of treatment, which can aid in avoiding adverse effects associated with orthodontic treatment. Some of the differences are caused by change in bone remodeling induced by drugs and systemic factors. ${ }^{3}$ All the drugs reviewed have therapeutic effects as well as side effects that influence the cells targeted by orthodontic forces. The value of a thorough medical history is increasingly significant as young and old alike are exposed to a greater range of therapeutic agents. Therefore, it is imperative that the orthodontists need to pay attention to drug consumption and history of each and every patient, before and during the course of orthodontic treatment, so that the best treatment strategy (including force control and appointment intervals) can be selected for each case. ${ }^{2}$

\section{Role of various drugs in orthodontic tooth movement:}

Drug molecules present in drugs and nutrients consumed regularly by patients can reach the mechanically stressed paradental tissues through the circulation and interact with local target cells. The combined effect of mechanical forces and one or more of these agents may be inhibitory, additive, or synergistic. Periodontal remodelling is a complex process regulated in parts by prostaglandins and adversely affected by the use of nonsterioidal antiinflammatory drugs. Orthodontic mechanical forces produce inflammation in periodontal tissues. Prostaglandins (PGs), lipid mediators derived from arachidonic acid (AA), play central roles in the pathogenesis of inflammation, fever, and pain. Evidence suggests that tooth movement significantly increased with prostaglandin injections. PGs are generated by the oxygenation of AA to the unstable intermediate prostaglandin H2 (PGH2) by PGHS, of which there are two major isoforms - the constitutive PGHS-1 and the (generally) inducible PGHS-2. These enzymes are also commonly referred to as cyclooxygenase (COX) 1 and 2 , respectively. ${ }^{4}$

Steroidal anti-inflammatory drugs such as glucocorticoids are indicated for the treatment of inflammatory disorders such as allergies, asthma, autoimmune diseases, and sepsis. Their efficacy in alleviating inflammatory disorders results from the pleiotropic effects of the glucocorticoid receptor on multiple signaling pathways.

Glucocorticoids are known to inhibit PG production through three independent mechanisms: suppression of signal transduction relating to proinflammatory cytokines, suppression of COX, and the activation of annexin I. Annexin I inhibits phospholipase A2_ (cPLA2), which results in the suppression of AA and its subsequent conversion to eicosanoids (ie, prostaglandins,thromboxanes, prostacyclins, and leukotrienes). Among the investigated drugs, celecoxib and prednisolone suppressed tooth movement and root resorption. Though the mechanism for suppression of root resorption is totally unknown, it may differ from the mechanism for suppression of tooth movement. Celecoxib and prednisolone may have an inhibitory effect on osteo/odontoclastic activity. The threshold dose for celecoxib to initiate the odontoclastic activity may be higher than that to initiate osteoclastic activity. ${ }^{5}$

Routinely the clinician prescribes the drugs for orthodontic pain. These drugs alter or interfere with the inflammatory process and therefore have an effect on the tooth movement. Several studies have proposed the effect of short and long term administration of medication on orthodontic tooth movement. Davidovitch et al. and Yamasaki et al. concluded in their study that the rate of orthodontic tooth movement can be altered by administrating certain drugs locally or systemically. The drugs used in orthodontics can be broadly classified into two major groups, promoter drugs and suppressor agents. Promoter drugs are agents that act with the secondary and primary inflammatory mediators and enhance the tooth movement, examples being; Prostaglandin, Leukotrienes, Cytokines, Vitamin D, Osteocalcin, and Corticosteroids. Suppressor agents are drugs which reduces bone resorption examples are; Nonsteroidal anti-inflammatory agents and bisphosphonates. the use of over-the-counter nonsteroidal anti-inflammatory drugs during tooth movement may result in aberrant remodeling of periodontal vasculature and other structures, ultimately affecting orthodontic treatment efficacy. The use of analgesics such as nonsteroidal antiinflammatorydrugs (NSAIDs) that inhibit the release of prostaglandin (PGs) and stop inflammation are effective in the treatment of pain related to orthodontic treatment. Nevertheless, the extended use of NSAIDs is inappropriate for orthodontic discomfort, because, as research and clinical experience suggests, their use could slow down tooth movement. NSAIDs are a chemically heterogeneous group of compounds, which nevertheless share certain therapeutic actions and adverse effects. The class includes derivatives of salicylic acid (e.g., aspirin, diflusinal), propionic acid (e.g., naproxen, ibuprofen, flurbiprofen, ketoprofen), acetic acid (e.g., indomethacin, etodolac, diclofenac, ketorolac), enolic acid (e.g., piroxicam, phenylbutazone), fenamic acid (e.g., mefenamic acid, meclofenamic acid), alkanones (nabumetone), and diaryl heterocyclic compounds (e.g., celecoxib, valdecoxib, rofecoxib, etoricoxib). ${ }^{6}$ 


\section{Root resorption by orthodontic tooth movement:}

Biomechanical treatment factors such as magnitude, duration, direction, and type of force (e.g.intermittent, interrupted, and continuous) can have an impact on root resorption. Root resorption is the progressive loss of dentine and cementum through the continued action of osteoclastic cells. Orthodontic tooth movement is possible as cementum has greater resistance to resorption than bone. However, root resorption is also seen after the application of light orthodontic force even within a short-duration period. In the early stages of treatment, this causes crown buccal and root lingual tipping of premolars leading to opposing areas of tension and compression in the crown and root. It is important to know that even in early stages of orthodontic treatment there is evidence of cemental resorption, which may not always be evident on radiographs unless it reaches an advanced stage. A radiographic examination is an essential part of the diagnostic process in orthodontics. Most clinicians order panoramic or periapical radiographs in addition to the cephalometric radiograph. Multiple theories have been postulated but chemical signals: osteoclast differentiation factor/osteoprotegrin ligand/receptor activator of nuclear factor $\kappa-\mathrm{B}$ ligand (ODF/OPGL/RANKL), are now known to control their formation. ${ }^{7}$

These are members of the tumour necrosis factor cytokine family. RANKL is liberated from osteoblasts and stromal cells. RANKL receptors are found on the surface of monocytes and macrophages and release is thought to stimulate these macrophages and mononuclear cells to fuse and become osteoclasts. RANKL is essential in both the development and function of osteoclasts. The osteoprotegrin/osteoclast inhibitory factor (OPG/OCIF) is a glycoprotein and is also a member of the tumour necrosis factor receptor super family. This too is secreted by osteoblasts and stromal cells but inhibits RANKL, thus inhibiting osteoclastic activity. RANKL expression can be stimulated by parathyroid hormone, vitamin D3 and interleukin-1B. Resorption has been reported in 19-31.4\% of all patients undergoing orthodontic treatment, its prevalence being highest in mandibular and maxillary incisors, with molar and canines being least effected. In 1991 Linge and Linge reported up to $2.5 \mathrm{~mm}$ of root reduction after orthodontic treatment. One meta-analysis has indicated the apical distance moved and duration of orthodontic treatment iscorrelated to the mean apical resorption. When occurring in adult patients it maybe more extreme. Ankylosis does not tend to occur with orthodontic related resorption and the periodontal ligament remains intact. Maintenance of vitality in orthodontically treated teeth is variable. ${ }^{8}$

Genetic tendencies and history of trauma appear to be most often associated with this pattern of resorption. This type of resorption has been named periapical replacement resorption (PARR) These include Substance P, calcitonin generelated peptide, neuropeptide $\mathrm{Y}$ and neurokinin $\mathrm{A}$. These are prominent mediators of increased vascularity locally. Such changes in vascularity may be linked to osteoclastic activity by speeding recruitment of inflammatory cells. In the PDL of heavily applied orthodontic rat molars, foci of lymphocytic infiltration were observed, which reflects inflammatory responses to applied orthodontic forces. The discontinuation of or reductions in orthodontic forces can stop root resorption and initiate the healing process in the cementum. Minor cemental tears are corrected by anatomic repair property of cellular cementum at the apex of the tooth. In rats, matrix metalloproteinase-1 (MMP-1) and cathepsin $\mathrm{K}$ are important in root resorption during tooth movement because they degrade the collagenous bone matrix in a mode similar to bone resorption. MMP-1 mRNA was detected in fibroblastic cells, cementoblasts, and osteoblasts but not in odontoclasts or osteoclasts. ${ }^{9}$ Moreover, MMP-1 mRNA was highly expressed in some cementocytes located near odontoclasts and in many osteocytes. On the other hand, cathepsin K mRNA was expressed only in odontoclasts and osteoclasts . Pro-inflammatory cytokines, such as interleukin (IL)-1beta, IL-2, IL-5, IL-6, IL-8, TNFalpha, interferon-gamma and GM-CSF, induce the classical hallmarks of inflammation through vasodilation and tissue invasion by leukocytes; whereas anti-inflammatory cytokines, such as IL-4 and IL10 , are involved in its resolution. Even systemic condition such as obesity is also known to influence systemic bone metabolism through complex mechanical, hormonal, and inflammatory interactions (López-Gómez et al., 2016) with associations between obesity and reduced bone remodelling (Ivaska et al., 2016) and increased bone mineral density (Salamat et al., 2016). Although there is little data relating obesity to changes in alveolar bone composition within the healthy periodontium, longitudinal data has shown a significant association with increased rates of tooth eruption (Must et al., 2012). Other studies reported that obesity may be influenced by the pathology caused by oral bacteria that increase metabolic efficiency and appetite, and redirect the metabolism of energy through increasing levels of TNF- $\alpha$.(Goodson et al., 2009). Moreover, inflammatory cytokines such as TNF and IL-1 are produced by adipocytes and lead to the pro-inflammatory state associated with obesity (Fantuzzi, 2005). Conversely, a significant reduction of IL- 6 and TNF- $\alpha$, coupled by an increase in IL-8 levels in the plasma, has been identified with weight loss (Bruun et al., 2003). ${ }^{10}$ A review of various biomarkers found in GCF and saliva with their functions and alterations in their levels due to various drug interactions has been summarized. ( Table 1) 


\section{Conclusion:-}

Understanding the biology of tooth movement and treatment outcomes individually is a complex process that requires knowledge in many different areas of biomedicine. The rapid development of molecular biology along with translational studies in humans and experimental systems are likely to provide us with a much more thorough insight into the cellular and molecular mechanisms involved in the bone remodeling processes induced by orthodontic forces. This is a prerequisite to understand the responses in different individuals and to develop new mechanisms by which tooth movement could be regulated not only by mechanical forces, but also by biological agents, if needed. Current researches tend to focus on areas such as monitoring patient's reaction to mechanical forces by searching bone remodeling markers in the GCF, saliva, and blood serum.

Table 1:- Various biomarkers found in GCF and saliva with their functions and alterations in level following particular drug interactions:

\begin{tabular}{|c|c|c|c|}
\hline Category & Marker & Function & Effect of drug intervention \\
\hline \multirow[t]{3}{*}{ Gcf Markers } & $\begin{array}{l}\text { PGE2, SubstanceP, } \\
\text { Interleukin } 1 \beta, 2,6 \text { and } \\
8, \text { Acid Phosphatase, } \\
\text { MMP } 1,2,3,8 \text { and 9, } \\
\text { Dentin Matrix Protein }\end{array}$ & Bone resorption & $\begin{array}{l}\text { 1. Salicylate therapy decreases bone resorption } \\
\text { by inhibition of PGs' synthesis and may effect } \\
\text { differentiation of osteoclasts from their } \\
\text { precursors. } \\
\text { 2. The levels of matrix metalloproteinases } \\
\text { (MMP9 and MMP2) were found to be } \\
\text { increased, along with elevated collagenase } \\
\text { activity, followed by a reduction in } \\
\text { procollagen synthesis which is essential for } \\
\text { bone and periodontal remodeling. The whole } \\
\text { process is controlled by inhibition of } \\
\text { cyclooxygenase (COX) activity, leading to } \\
\text { altered vascular and extravascular matrix } \\
\text { remodeling, causing a reduction in the pace of } \\
\text { the tooth movement. }\end{array}$ \\
\hline & $\begin{array}{l}\text { Transforming Growth } \\
\text { Factor } \beta, \text { RANK-L, } \\
\text { Osteoprotegrin, } \\
\text { Osteocalcin, } \\
\text { Pyridinoline, Alkaline } \\
\text { Phosphatase }\end{array}$ & $\begin{array}{l}\text { Bone } \\
\text { remodeling }\end{array}$ & $\begin{array}{l}\text { 1. The appearance of osteoclasts in the } \\
\text { interradicular septum of bone of the first molar } \\
\text { was found to be inhibited by the indomethacin. } \\
\text { vitamin D metabolite, 1,25-dihydroxy } \\
\text { cholecalciferol, caused increase in the number } \\
\text { of osteoclasts and amount of tooth movement } \\
\text { during canine retraction with light forces. } \\
\text { 2. Sodium fluoride has been shown to inhibit the } \\
\text { osteoclastic activity and reduce the number of } \\
\text { active osteoclasts. } \\
\text { 3. Bisphosphonates cause a rise in intracellular } \\
\text { calcium levels in osteoclastic-like cell line, } \\
\text { reduction of osteoclastic activity, prevention of } \\
\text { osteoclastic development from hematopoietic } \\
\text { precursors, and production of an osteoclast } \\
\text { inhibitory factor }\end{array}$ \\
\hline & $\begin{array}{l}\text { Glycosaminoglycans } \\
\text { ( Hyluronic acid, } \\
\text { Chondroitin Sulfate), } \\
\text { Insulin like Growth } \\
\text { Factor, Aspartate } \\
\text { Aminotransferase, } \\
\text { Cathepsin, Lactate } \\
\text { Dehydrogenase }\end{array}$ & 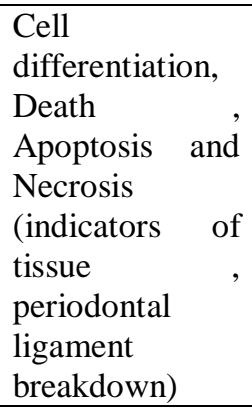 & $\begin{array}{l}\text { 1. Immunomodulatory drugs modulate nuclear } \\
\text { factor kappa - Beta, tyrosine kinases in } \\
\text { signaling pathway, IL-6, MMPs and PGE2. } \\
\text { 2. Corticosteroids increase the rate of tooth } \\
\text { movement, and since new bone formation can } \\
\text { be difficult in a treated patient, they decrease } \\
\text { the stability of tooth movement and stability of } \\
\text { orthodontic treatment in general. }\end{array}$ \\
\hline Salivary/ Erum & Albumin, $\mathrm{C}$ reactive & & 1. IL antagonists inhibit IL-1, produced by \\
\hline
\end{tabular}




\begin{tabular}{|l|l|l|l|}
\hline Markers & $\begin{array}{l}\text { protein, IL-6, } \alpha 2 \\
\text { microglobulin, } \\
\text { Myeloperoxidase }\end{array}$ & $\begin{array}{l}\text { monocytes, macrophages, and some } \\
\text { specialized cells, which are important for the } \\
\text { inflammatory response, and IL-6 and COX-2. } \\
\text { These drugs influence the inflammatory } \\
\text { response following force application, reducing } \\
\text { the pace of tooth movement and bone } \\
\text { remodeling. }\end{array}$ \\
\hline
\end{tabular}

\section{References:-}

1. Alhadlaq AM. Biomarkers of Orthodontic Tooth Movement in Gingival Crevicular Fluid: A Systematic Review. J Contemp Dent Pract. 2015 Jul 1;16(7):578-87

2. de Aguiar MC, Perinetti G, Capelli J Jr. The Gingival Crevicular Fluid as a Source of Biomarkers to Enhance Efficiency of Orthodontic and Functional Treatment of Growing Patients. Biomed Res Int. 2017;2017:3257235. doi:10.1155/2017/3257235.

3. Kumar A A, Saravanan K, Kohila K, Kumar S S. Biomarkers in orthodontic tooth movement. J Pharm Bioall Sci 2015;7, Suppl S2:325-30.

4. Kapoor P, Monga N, Kharbanda OP, Kapila S, Miglani R, Moganty R. (2019). Effect of orthodontic forces on levels of enzymes in gingival crevicular fluid (GCF): A systematic review. Dental Press Journal of Orthodontics, 24(2), 40.e1-40.e22.

5. Gaur A, Maheshwari S, Verma SK. Detection of molecular biomarkers as a diagnostic tool in the planning and progression of orthodontic treatment. J Orofac Sci 2017;9:80-4.

6. Roohi A, Tabrizi M, Abbasi F, Ataie-Jafari A, Nikbin B, Larijani B, Qorbani M, Meysamie A, Asgarian-Omran H, Nikmanesh B, Bajouri A, Shafiey N, Maleki A. Serum IL-17, IL-23, and TGF- $\beta$ levels in type 1 and type 2 diabetic patients and age-matched healthy controls. Biomed Res Int. 2014;2014:718946. doi: 10.1155/2014/718946. Epub 2014 Jun 4. PMID: 24995325; PMCID: PMC4065742.

7. Yadav $\mathrm{H}^{1}$, Quijano C, Kamaraju AK, Gavrilova O, Malek R, Chen W, Zerfas P, Zhigang D, Wright EC, Stuelten C, Sun P, Lonning S, Skarulis M, Sumner AE, Finkel T, Rane SG. Protection from obesity and diabetes by blockade of TGF- $\beta / S m a d 3$ signaling. Cell Metab. 2011 Jul 6;14(1):67-79. doi: 10.1016/j.cmet.2011.04.013.

8. Kouskoura T, Katsaros C, von Gunten S. The Potential Use of Pharmacological Agents to Modulate Orthodontic Tooth Movement (OTM). Front Physiol. 2017 Feb 8;8:67. doi: 10.3389/fphys.2017.00067. PMID: 28228735; PMCID: PMC5296343.Diravidamani K, Sivalingam SK, Agarwal V. Drugs influencing orthodontic tooth movement: An overall review. J Pharm Bioallied Sci. 2012 Aug;4(Suppl 2):S299-303. doi: 10.4103/09757406.100278. PMID: 23066275; PMCID: PMC3467877.

9. B. Deschner, B. Rath, A. Jäger, J. Deschner, B. Denecke, S. Memmert, and W. Götz, "Gene analysis of signal transduction factors and transcription factors in periodontal ligament cells following application of dynamic strain," Journal of Orofacial Orthopedics / Fortschritte der Kieferorthopädie, vol. 73, no. 6, pp. 486-497, 2012. 